\title{
Prevencion de Riesgos en la Universidad de Granada
}

\author{
Espinosa Hidalgo, Pedro; Villalba Moreno, Juan; \\ Machado Santiago, Javier; Rodriguez Tapioles, Reyes; \\ Muros Ladrón de Guevara, Juan; Garcia Rodriguez, Elias \\ Gabinete de Prevención y Calidad Ambiental \\ Universidad de Granada
}

La Universidad de Granada ha creado el Gabinete de Prevención y Calidad Ambiental para la respuesta a los retos planteados en el campo de la prevención de riesgos laborales y la protección del medio ambiente.

En éste artículo se describen cómo se ha organizado (funciones, estructura, personal, etc), los tres servicios de los que está compuesto éste Gabinete (Servicio de Prevención de Riesgos Laborales, Servicio de Protección Radiológica y Unidad de Calidad Ambiental), así como la descripción y primeras actuaciones del órgano de participación y consulta que prevé la ley de Riesgos Laborales, que es el Comité de Seguridad y Salud de la Universidad de Granada.

The University of Granada has founded an Office for Prevention and Environmental Quality to meet the challenges set by the prevention of work risks and environment protection.

In this article we describe how the three services of the Cabinet (Service for the Protection of Work Risks, Service for Radiological Protection, Environmental Quality Unit) are organised (functions, structure, workforce, etc). We also include a description and the first accomplishments of the participation and consulting body in accordance with the Law on Risk Prevention in the Workplace, called the Committee for Health and Security of the University of Granada.

Palabras clave: Prevención, calidad ambiental, universidad de Granada

Key words: Prevention, environmental quality, University of Granada

\section{Comite de Seguridad y SALUd}

La estructura de participación en materias de seguridad y salud laboral de la Universidad de Granada (UGRA) se recoge en la figura 1. 


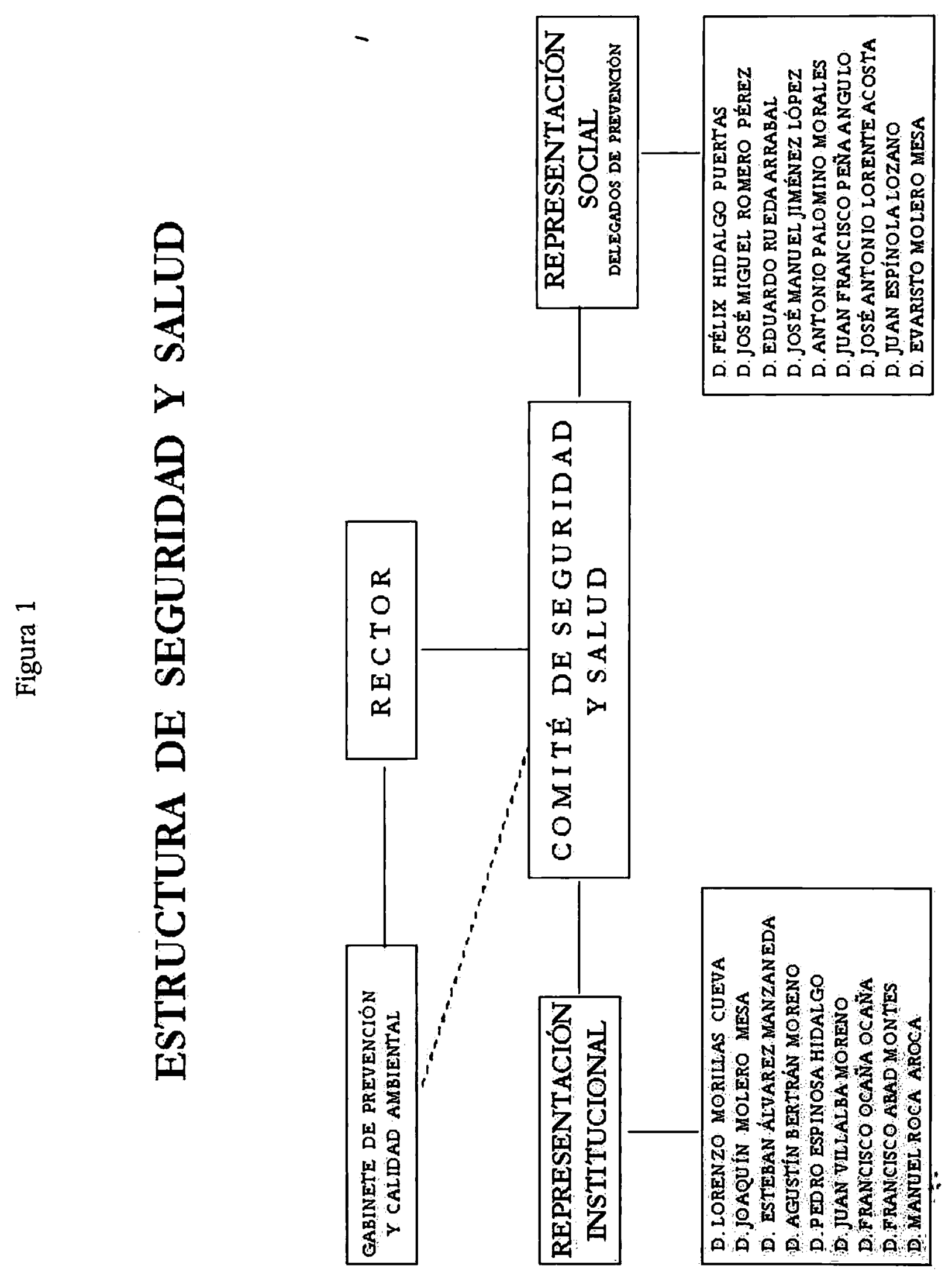


El Comité de Seguridad y Salud (CSS) de la Universidad de Granada (UGRA) es el órgano paritario y colegiado de participación destinado a la consulta regular y periódica de las actuaciones en materia de prevención de riesgos laborales. Dicho comité fue constituido en febrero de 1997.

El CSS abordó en sus primeras sesiones los siguientes temas:

* Elaboración del reglamento interno.

* Creación de una comisión encargada de elaborar una relación de las actuaciones más urgentes en materia de prevención de riesgos.

* Dotación de un presupuesto adecuado para ir acometiendo las actuaciones planificadas.

* Elaboración del Proyecto del Plan de Prevención de Riesgos Laborales de la Universidad de Granada.

\subsection{COMPOSICIÓN DEL CSS DE LA UGRA}

El CSS está integrado por dieciocho miembros:

a) Nueve representantes de la Administración Universitaria designados por el Rector por períodos de cuatro años.

b) Nueve delegados de prevención.

Son elegidos por los órganos de representación conforme a la normativa vigente y a acuerdos específicos, con la periodicidad establecida para las elecciones a dichos órganos.

El delegado de prevención que pierda la condición de miembro del Comité de Empresa o de cualquiera de las Juntas de Personal, perderá igualmente la condición de miembro del CSS.

El CSS está presidido por el Rector de la Universidad, quien podrá delegar en la autoridad académica que designe.

La designación del Secretario se realiza por y entre los delegados de prevención, así como el cese o la sustitución temporal en los casos de vacante, ausencia o enfermedad.

La composición actual del CSS se recoge en la figura 1.

El CSS cuenta también con la participación, con voz pero sin voto, de los delegados sindicales y del personal técnico del Servicio de Prevención y, en función de los temas tratados, está abierto a la participación de cualquier otra persona que el propio Comité estime oportuna. 


\subsection{COMPETENCLAS DEL CSS}

- Participar en la elaboración, puesta en práctica y evaluación de los planes y programas de prevención de riesgos en la UGRA.

- Promover iniciativas sobre métodos y procedimientos para la efectiva prevención de los riesgos, proponiendo la mejora de las condiciones o la corrección de las deficiencias existentes.

- Cualesquiera otras que se establezcan en las leyes.

\subsection{FACUlTADES DEL. CSS.}

- Conocer directamente la situación relativa a la prevención de riesgos en el centro de trabajo, realizando a tal efecto las visitas que estime oportunas.

- Conocer cuantos documentos e informes relativos a las condiciones de trabajo sean necesarios para el cumplimiento de sus funciones, así como los procedentes de la actividad del Servicio de Prevención, en su caso.

- Conocer y analizar los daños producidos en la salud o en la integridad física de los trabajadores, valorar sus causas y proponer las medidas preventivas oportunas.

- Conocer e informar la memoria y programación anual del Servicio de Prevención.

El reglamento del CSS, que fija las bases de su actuación, algunas de las cuales hemos señalado antes, fue aprobado en el mes de junio del año 1997. Prevé como mínimo una reunión ordinaria cada tres meses y reuniones extraordinarias por iniciativa del Presidente o por petición fundada de un tercio de los miembros.

\subsection{COMISIÓN DEL CSS}

Para agilizar las actuaciones propias del CSS entre reuniones, en febrero de 1999 se constituyó una Comisión del CSS formada por 6 miembros, 3 vocales representando a la Institución y 3 vocales designados por y entre los Delegados de Prevención, siendo uno de ellos el Secretario del CSS.

La representación de la Institución la ostenta el Gerente, el Vicerrector de Infraestructuras y el Director del Gabinete de Prevención y Calidad Ambiental. La representación de los Delegados de Prevención la ostenta un representante poir 
cada uno de los tres sindicatos mayoritarios en la Universidad (CCOO, UGT Y CSIF).

Esta Comisión tiene previstas reuniones mensuales.

2. Organización de la prevención en la Universidad de Granada. Gabinete de Prevención y Calidad Ambiental

En octubre de 1997, la UGRA creó el Gabinete de Prevención y Calidad Ambiental (GPCA) siendo sus objetivos proponer y desarrollar las actividades preventivas relativas a los riesgos laborales y medio ambientales.

Para ello, se le dotó de la estructura organizativa apropiada que le permitiese planificar y coordinar las actividades de prevención (figura 2), y se le asignó un presupuesto específico para el desarrollo de sus funciones, que en la actualidad es de 50 millones de pesetas.

Figura 2

\section{ORGANIZACIÓN DE LA PREVENCIÓN}

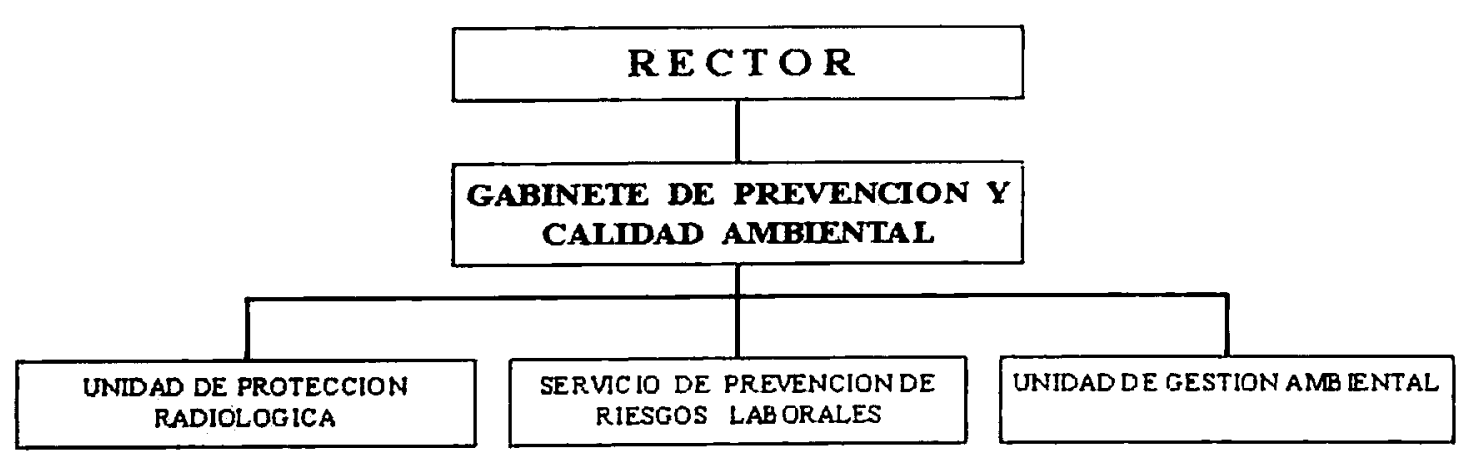

A continuación haremos una breve descripción de las actividades desarrolladas por cada una de las unidades que componen el GPCA y de los programas que tienen previsto desarrollar en los próximos años. 
3. Servicio de Prevención de Riesgos laborales

En la figura 3 se recoge la composición por áreas del Servicio de Prevención. De las cuatro áreas de que consta, en la actualidad solamente está dotada el área de Salud. Está previsto cubrir próximamente las áreas de Seguridad e Higiene.

Figura 3

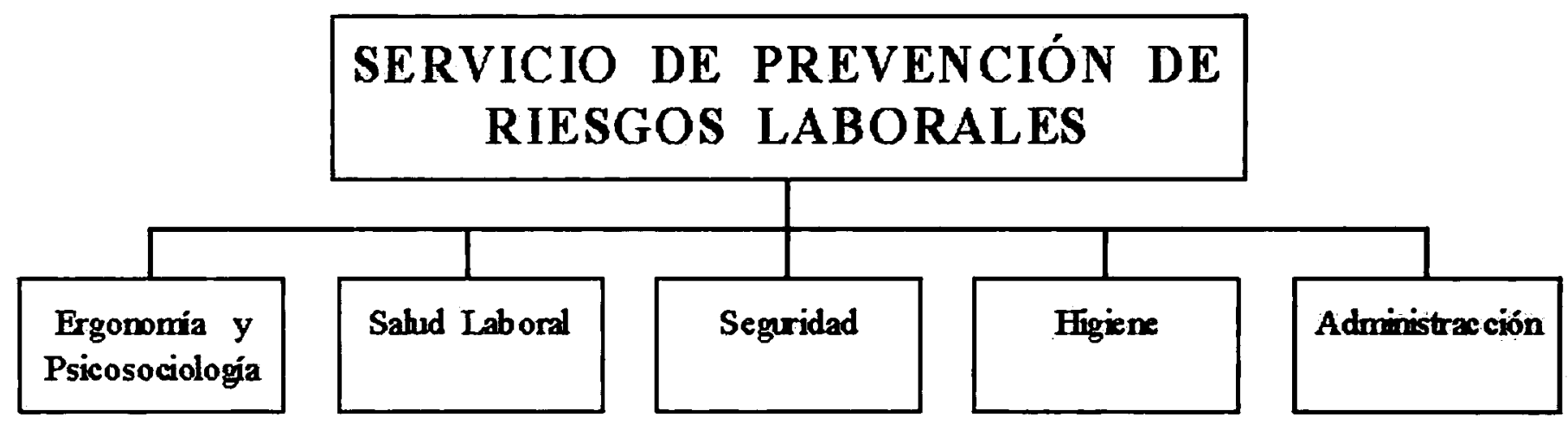

\subsection{Medios Humanos}

El Servicio de Prevención está dotado de dos médicos especialistas en Medicina del Trabajo, uno de los cuales simultanea funciones de Medicina Laboral con las de Higiene, y dos ATS-DUE de empresa, uno de los cuales simultanea sus funciones con las de Técnico de Seguridad. Las tareas de administración las realizan dos administrativos.

\section{2. actividades del Servicio de Prevención}

En las tablas 1 y 2 se recogen las actividades llevadas a cabo por el Servicio de Prevención desde su creación.

Los reconocimientos médicos se han priorizado por grupos de riesgo laboral: exposición a tóxicos, exposición a radiaciones ionizantes, contaminaciốn biológi$\mathrm{ca}$, manejo de cargas y sobreesfuerzos, uso de pantallas de visualización de datós, etc. 
Tabla 1. Actividades realizadas en materia de Salud

\begin{tabular}{|c|c|c|c|c|}
\hline \multicolumn{5}{|c|}{ AREA DE SAIUD } \\
\hline $\begin{array}{l}\text { PROGRAMA DE PREVENCIÓN Y } \\
\text { PROMOCION DE LA SALUD }\end{array}$ & $94-95\left(^{\circ}\right)$ & $1996\left({ }^{\circ}\right)$ & 1997 & 1998 \\
\hline $\begin{array}{l}\text { - PERSONAL EXPUESTO A P.V.D. } \\
\text { RECONOCIMIENTOS PERIODICOS. } \\
\text { - RECONOCIMIENTOS POR CAMBIOS DE PUIESTO. } \\
\text { RECONOCIMIENTOS DE INCAPACIDAD TEMPORAL. } \\
\text { - RECONOCIMIENTOS A PETICION DEL TRABAJADOR. } \\
\text { - RECONOCIMIENTOS PREVIOS AL INGRESO. } \\
\text { - RECONOCIMIENTOS A PERSONAL EXPUESTO A } \\
\text { RADIACIONES IONIZANTES. }\end{array}$ & $\begin{array}{r}- \\
688 \\
37 \\
31 \\
- \\
- \\
-\end{array}$ & $\begin{array}{r}- \\
421 \\
9 \\
190 \\
42 \\
12 \\
16\end{array}$ & $\begin{array}{r}502 \\
7 \\
270 \\
29 \\
39 \\
22\end{array}$ & $\begin{array}{r}531 \\
8 \\
108 \\
10 \\
91 \\
29\end{array}$ \\
\hline PROGRAMA SANITARIO & $94-95(*)$ & $1996(\bullet)$ & 1997 & 1998 \\
\hline $\begin{array}{l}\text { - CONSULTAS MÉDICAS. } \\
\text { INMUNIZACIONES } \\
\text { VACUNACIÓN ANTIGRIPAL. } \\
\text { VACUNACION ANTITETANICA. } \\
\text { VACUNACIÓN ANTIHEPATITIS B. } \\
\text { CURAS DE ENFERMERIA. } \\
\text { - REPOSICION DE BOTIQUINES. }\end{array}$ & $\begin{array}{r}60 \\
770 \\
981 \\
596 \\
36 \\
50\end{array}$ & $\begin{array}{r}166 \\
327 \\
856 \\
473 \\
58 \\
82\end{array}$ & $\begin{array}{r}127 \\
367 \\
715 \\
173 \\
88 \\
86\end{array}$ & $\begin{array}{r}100 \\
348 \\
664 \\
164 \\
64 \\
98\end{array}$ \\
\hline PROGRAMA DE HIPERTENSIÓN & $94-95(\cdot)$ & $1996\left(\bullet^{\circ}\right)$ & 1997 & 1998 \\
\hline - TOMA Y CONTROL DE TENSIÓN ARTERIAL. & 240 & 323 & 434 & 442 \\
\hline
\end{tabular}

(*) Durante estos años funcionaba el Servicio de Salud Laboral, antecedente del Servicio de Prevención que se constituyó con tal denominación en el año 1997. 
Tabla 2. Actividades realizadas en materia de seguridad

\begin{tabular}{|l|r|r|r|}
\hline \multicolumn{3}{|c|}{ ÁREA DE SEGURIDAD } \\
\hline \multirow{2}{*}{ ACTUACIONES } & \multicolumn{3}{c|}{ INVERSIONES } \\
\cline { 2 - 5 } & $1996-1997$ & 1998 & 1999 \\
\hline SEÑALIZACIÓN SEGURIDAD/EVACUACION & 1004047 & 1801592 & 3529228 \\
\hline DOTACION Y MANTENIMIENTO DE EXIINTORES & 2297264 & 340244 & 494679 \\
\hline PUERTAS CORTAFUEGOS & 1570100 & - & - \\
\hline SISTEMAS DE ALARMA Y DETECCION DE INCENDIOS & 956342 & - & 195298 \\
\hline RAMPAS MIINUSVÁIIDOS & 162800 & - & - \\
\hline ADAPTACIÓN PUERTAS DE SEGURIDAD Y AULAS & 650710 & - & - \\
\hline EQUIPOS DE PROTECCIÓN INDIVIDUAI (EPIS) & - & 146438 & 134999 \\
\hline OTROS (ASCENSORES,...) & - & - & 538280 \\
\hline
\end{tabular}

\subsection{Planificación y programación}

A continuación comentaremos las acciones fundamentales que tiene previsto realizar el SPRL de la UGRA durante los años 1999 y 2000.

\subsubsection{Valoración de la situación actual. Evaluación inicial de riesgos}

Para establecer la situación de partida en materia de condiciones de trabajo, accidentes laborales, grado de control y peligrosidad de los riesgos laborales en la UGRA estamos realizando actualmente la evaluación inicial de riesgos.

El art. 15 de la Ley de Prevención de Riesgos Laborales (LPRL) establece come principios de la acción preventiva, en primer lugar evitar riesgos, y, en segundo lugar, evaluar los riesgos que no se pueden evitar. Los arts. 4 al 7 establecen como debe planificarse dicha evaluación.

El art.4 del Reglamento de los Servicios de Prevención establece que para realizar la evaluación de riesgos deben tenerse en cuenta:

a) Las condiciones de trabajo existentes o previstas en cada puesto. 
b) La especial sensibilidad de los trabajadores que los ocupan o vayan a ocuparlos.

La LPRL define condición de trabajo como "cualquier característica del mismo que puede tener una influencia significativa en la generación de riesgos para la seguridad y salud del trabajador".

Las características incluidas en esta definición son:

a) Las características generales de los locales, instalaciones, equipos productos y demás útiles existentes en el centro de trabajo.

b) La naturaleza de los agentes físicos, químicos y biológicos presentes en el ambiente de trabajo y sus correspondientes intensidades, concentraciones o niveles de existencia.

c) Todas aquellas otras características del trabajo, incluidas las relativas a su organización y ordenación, que influyan en la magnitud de los riesgos a que esté expuesto el trabajador.

En la UGRA hemos planificado la evaluación de riesgos en dos fases (figura 4):

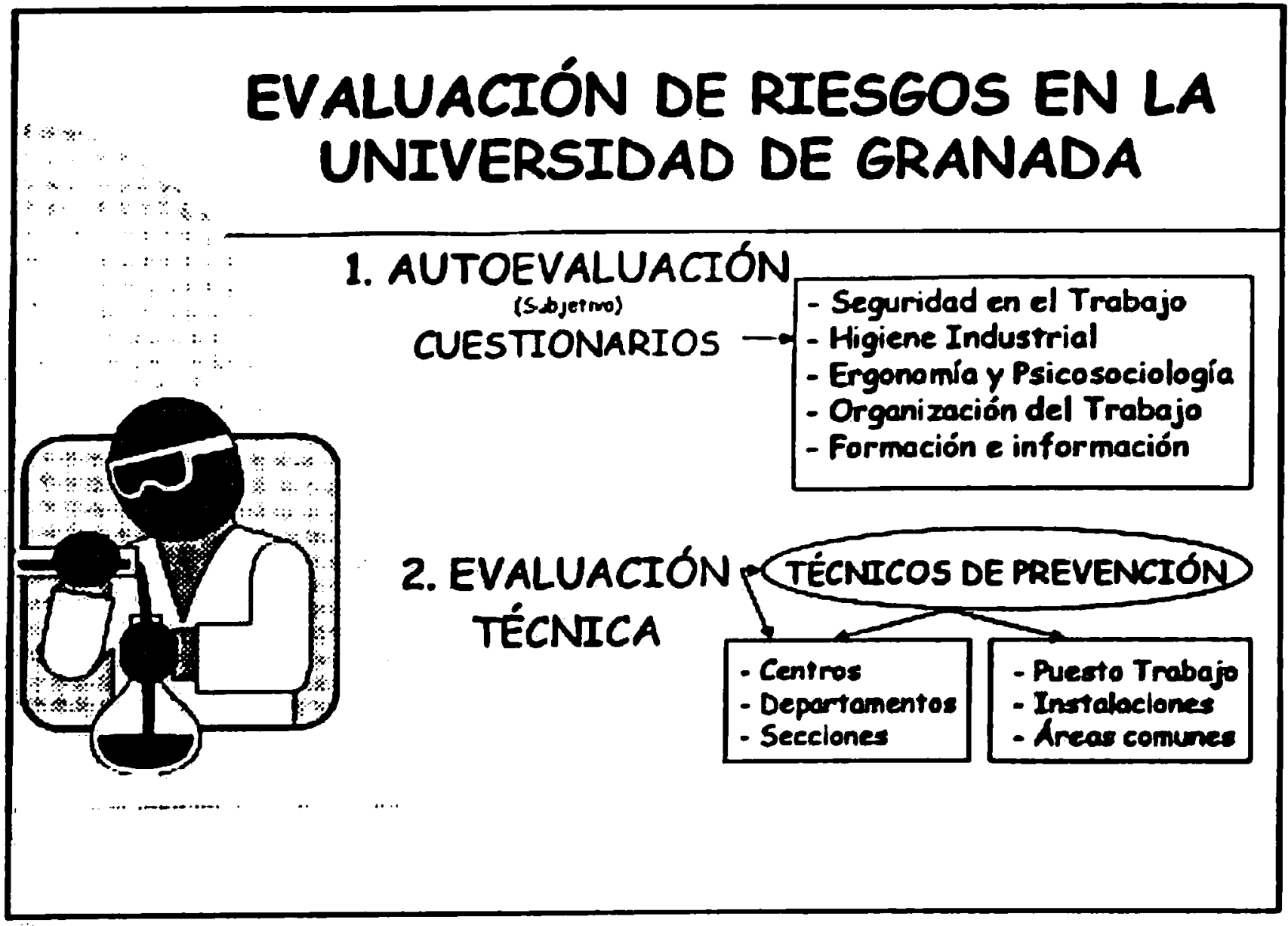


La primera fase, consiste en una autoevaluación por parte de cada trabajador de sus condiciones de trabajo. Con ello perseguimos, además de los objetivos generales enunciados anteriormente, la participación e integración de los trabajadores así como una labor inicial informativa.

1. En esta fase se están utilizando unos cuestionarios identificados. Dichos cuestionarios contienen preguntas cuya respuestas son, fundamentalmente, del tipo $\mathrm{si} / \mathrm{no}$; otras preguntas se responden con referencia a una escala de valoración.

2. La segunda fase consiste en una evaluación técnica por puestos de trabajo, por secciones (departamentos o servicios) y centros. Será realizada por el personal técnico del Servicio de Prevención y en ella se tendrá en cuenta la información aportada por la primera fase.

Las condiciones de trabajo evaluadas en ambas fases son las que, clasificadas en cinco grupos, se recogen en la tabla 3.

Tabla 3. Evaluación inicial de riesgos: Condiciones de trabajo evaluadas

\begin{tabular}{|c|c|c|c|c|c|}
\hline \multirow{2}{*}{ Código } & \multirow{2}{*}{ Evaluación DE RIESGos } & \multicolumn{3}{|c|}{ Técnica } & \multirow{2}{*}{ Individual } \\
\cline { 3 - 5 } & & Indiv. & Secci. & Centrovalu) \\
\cline { 3 - 4 } & Gestión Preventiva & & & & \\
\hline
\end{tabular}

\begin{tabular}{|c|c|c|c|c|c|}
\hline \multirow[t]{2}{*}{ Código } & \multirow[t]{2}{*}{ EVALUACIÓN DE RUESGOS } & \multicolumn{3}{|c|}{ Técnica } & \multirow{2}{*}{$\frac{\text { Individual }}{\text { (Autoevalu) }}$} \\
\hline & & Indiv. & Secci. & Centro & \\
\hline 2.1 & Lugares de trabajo & $\mathbf{x}$ & $\mathbf{x}$ & & $\mathbf{x}$ \\
\hline 2.2 & Máquinas & $\mathbf{x}$ & $\mathbf{x}$ & $\mathbf{x}$ & \\
\hline 2.3 & Elevación y transporte & & & $\dot{x}$ & \\
\hline 2.4 & Herramientas manuales & $\mathbf{x}$ & & & $\mathbf{x}$ \\
\hline 2.5 & Manejo manual de cargas & $\mathbf{x}$ & & & $x$ \\
\hline 2.6 & Instalaciones eléctricas & $\mathbf{x}$ & $x$ & $\mathbf{x}$ & \\
\hline 2.7 & Aparatos a presión y gases & $\mathbf{x}$ & $\mathbf{x}$ & $\mathbf{x}$ & $\therefore$ \\
\hline 2.8 & Protección contra incendios & & $\mathbf{x}$ & $\mathbf{x}$ & $\mathbf{x}$ \\
\hline 2.9 & Sustancias químicas & $\mathbf{x}$ & $\mathbf{x}$ & & $\mathbf{x}$ \\
\hline 2.10 & Accidentabilidad & $\mathbf{x}$ & & & $\mathbf{x}$ \\
\hline 2.11 & Equipos de protección individual & $\mathbf{x}$ & $\mathbf{x}$ & & $\mathbf{x}$ \\
\hline
\end{tabular}


Tabla 3 (continuación).

\begin{tabular}{|c|l|c|c|c|c|}
\hline \multirow{2}{*}{ Código } & EVALUACIÓN DE RIESGOS & \multicolumn{3}{|c|}{ Técnica } & \multicolumn{1}{c|}{$\begin{array}{c}\text { Individual } \\
\text { (Autoevalu.) }\end{array}$} \\
\hline 3.1 & & Indiv. & Secci. & Centro & $\mathrm{x}$ \\
\hline 3.2 & Contaminantes químicos & $\mathrm{x}$ & $\mathrm{x}$ & & $\mathrm{x}$ \\
\hline 3.3 & Ventaminantes biológicos & $\mathrm{x}$ & $\mathrm{x}$ & & $\mathrm{x}$ \\
\hline 3.4 & Ruido & & $\mathrm{x}$ & $\mathrm{x}$ & $\mathrm{x}$ \\
\hline 3.5 & Vibraciónes & $\mathrm{x}$ & & & $\mathrm{x}$ \\
\hline 3.6 & Iluminación & $\mathrm{x}$ & & & $\mathrm{x}$ \\
\hline 3.7 & Calor y frío & $\mathrm{x}$ & $\mathrm{x}$ & & $\mathrm{x}$ \\
\hline 3.8 & Radiaciones ionizantes & $\mathrm{x}$ & & & $\mathrm{x}$ \\
\hline 3.9 & Radiaciones no ionizantes & $\mathrm{x}$ & $\mathrm{x}$ & & $\mathrm{x}$ \\
\hline 3.10 & Trabajo con sustancias cancerigenas & $\mathrm{x}$ & & & \\
\hline
\end{tabular}

\begin{tabular}{|c|c|c|c|c|c|}
\hline Código & EVALUACION DE RIESGOS & \multicolumn{3}{|c|}{ Técnica } & \multirow{2}{*}{$\begin{array}{l}\text { Individual } \\
\text { (Autoevalu.) }\end{array}$} \\
\hline 4 & $\begin{array}{l}\text { Condiciones de Ergonomía } \\
\text { y psicosociologia }\end{array}$ & Indiv. & Secci. & Centro & \\
\hline 4.1 & Carga mental (P.A.S.) & & & & $\mathrm{x}$ \\
\hline 4.2 & Factores de estrés (Profesores) & & & & $\mathrm{x}$ \\
\hline 4.3 & Pantallas de visualización de datos & $\mathbf{x}$ & & & $\mathrm{x}$ \\
\hline 4.4 & Factores de organización & $\mathbf{x}$ & $\mathrm{x}$ & $\mathrm{x}$ & $\mathbf{x}$ \\
\hline
\end{tabular}

\begin{tabular}{|c|c|c|c|c|c|}
\hline Código & EVALUACIÓN DE RIESGOS & \multicolumn{3}{|c|}{ Técnica } & Ind \\
\hline 5 & Formación e información & Indiv. & Secci. & Centro & (Autoevalu.) \\
\hline 5.1 & Formación & $\mathbf{x}$ & & & $\mathbf{x}$ \\
\hline
\end{tabular}

Algunos cuestionarios son comunes para todo el personal universitario: 1, 2.1, 2.8, 2.10, 3.3, 4.1, 4.2 y 5.1. Los demás serán cumplimentados sólo por aquellos trabajadores según los riesgos específicos a los que estén expuestos (figura 5). 


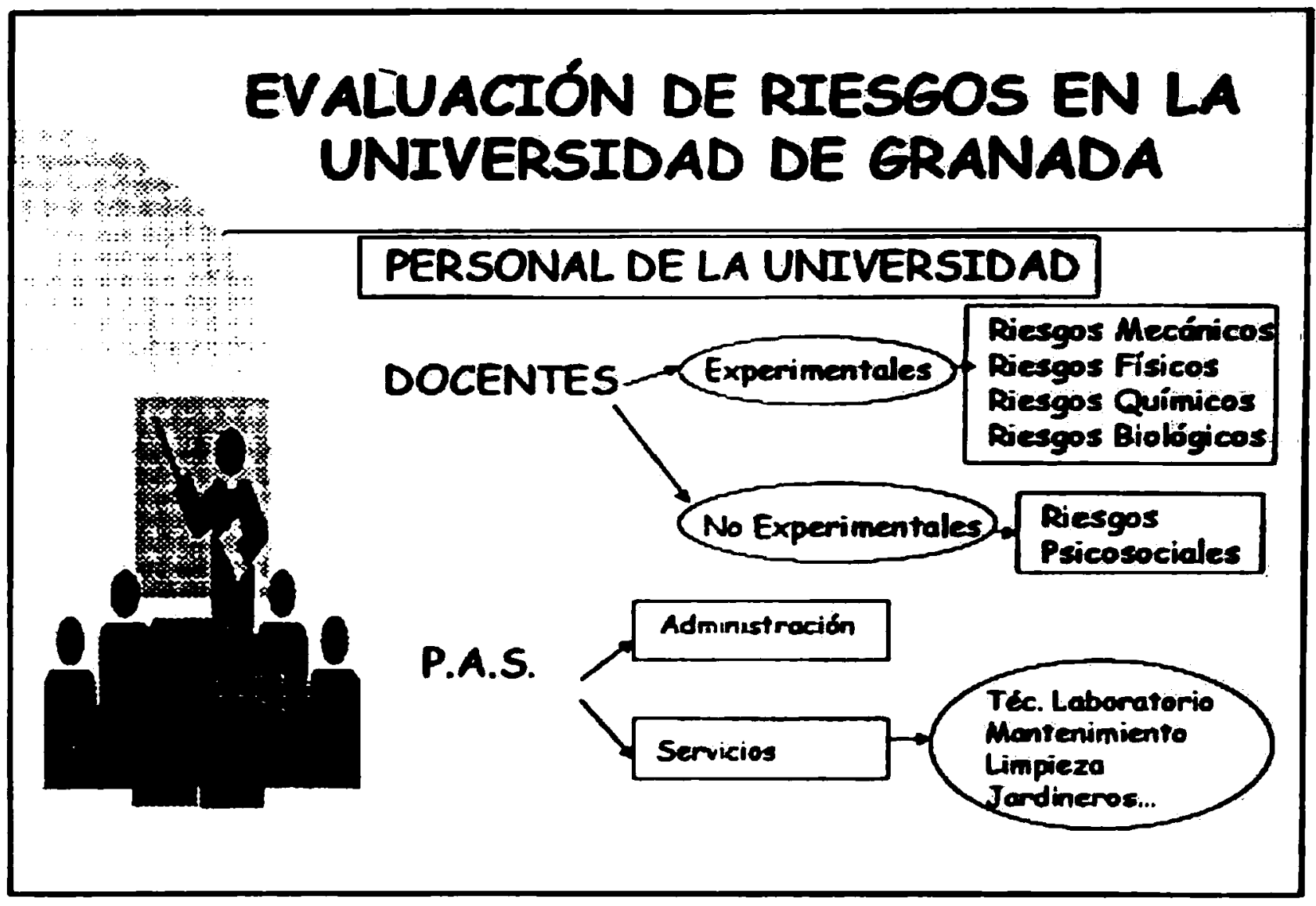

En el apartado de los factores psicosociales se ha distinguido entre el personal de administración y servicios y el profesorado. Para evaluar la carga mental del P.A.S. se ha utilizado el cuestionario del Instituto Nacional de Seguridad e Higiene en el Trabajo. Para evaluar la carga mental del profesorado se ha desarrollado un cuestionario especifico en colaboración con profesores del departamento de Psicología Social y Metodología de las Ciencias.

\subsubsection{Estadísticas de accidentes laborales}

Desde hace cinco años se vienen registrando los accidentes de trabajo del personal de administración y servicios (P.A.S) y profesores contratados (régimen general de la seguridad social). El profesorado funcionario (MUFACE) no ha comunicado durante este tiempo ningún accidente.

El registro aporta datos de una validez relativa debido a que el personal universitario no está habituado, en general, a dar parte de los accidentes de trabajo; fa veces por la sencilla razón que no tiene claro el concepto accidente de trabajo In este sentido parece extraño que ni el profesorado funcionario (1.900) ni el comtra- 
tado (600) hayan comunicado ningún accidente en este período. En lo que al PAS se refiere se vienen registrando del orden de 40 accidentes al año.

\subsubsection{Programas de prevención a desarrollar}

No obstante se pueden enumerar algunos de los programas que consideramos esenciales:

- Desarrollo de metodologías para llevar a cabo la evaluación inicial de riesgos y las más específicas de riesgos no controlados.

- Corrección de deficiencias técnicas catalogadas como urgentes.

- Programa de formación general y específica en función de los riesgos.

- Registro y protocolo de investigación de accidentes y enfermedades profesionales.

- Planificación de los controles periódicos de higiene y seguridad.

- Protocolos específicos para la vigilancia de la salud.

- Programas de emergencias y prevención de incendios por centros.

- Gestión de residuos peligrosos y radiactivos.

- Identificación y protección del personal especialmente sensible o expuesto a riesgos especiales.

\subsubsection{Formación de alumnos}

El CSS de la UGRA señaló entre los objetivos del Servicio de Prevención dar una formación adecuada a los alumnos en temas como la seguridad y el respeto al medio ambiente.

Entendemos que la conciencia de seguridad debe ser transmitida por el profesor o el técnico al alumno en todas las enseñanzas científicas impartidas en nuestra Universidad, aunque de manera especial en las prácticas de laboratorio.

La asimilación del concepto de seguridad debe contemplar tres fases, "antes", "durante" y "después" de las prácticas.

\section{Antes de las prácticas}

1. Edificios e instalaciones seguros.

2. Formación previa y continuada de docentes y técnicos de laboratorio.

3. Confección de protocolos de trabajo seguros.

\section{Durante las prácticas}

4. Información de los riesgos y de su prevención y control a los alumnos.

5. Utilización de medidas de seguridad colectivas e individuales. 
6. Gestión de los residuos en el centro.

\section{Después de las prácticas}

7. Orden y limpieza (para próximos usuarios del local).

8. Gestión de los residuos fuera del centro.

9. Proceso de mejora continuada.

Desde hace aproximadamente año y medio estamos incidiendo en las dos últimas fases. Para ello, se han impartido charlas de 1 hora de duración sobre seguridad en prácticas de laboratorio. En estas sesiones se les distribuye también trípticos con normas de seguridad y de comportamiento, y pegatinas con los pictogramas de peligrosidad de los productos químicos que van a manejar.

El problema principal que encontramos es el número de sesiones que deben impartirse para llegar a todos los alumnos en prácticas. Para intentar resolverlo, en el curso 1999-2000 tenemos prevista la formación de monitores entre alumnos de $4^{\circ}$ y $5^{\circ}$ de las licenciaturas, los cuales participarán, junto con los miembros del GPCA, en la labor de formación de todo el alumnado.

El cursillo de formación de monitores tiene las siguientes caracteristicas:

Número máximo de alumnos: 20

Duración: 30 horas

Contenido:

- Técnicas de docencia.

- Legislación en prevención de riesgos (conocimientos generales)

- Métodos de trabajo en el laboratorio.

- Toxicología básica.

- Señalización de seguridad. Etiquetado, clasificación y almacenamiento de productos químicos.

- Medidas colectivas de prevención en laboratorios.

- Equipos de protección individual.

- Trabajo con sistemas de gases a presión.

- Extintores de incendios.

- Gestión de residuos.

- Primeros auxilios.

- Diseño de las clases de prácticas.

- Evaluación.

\section{SERVICIO DE PROTECCIÓN RADIOLOGICA}

Las primeras instalaciones radiactivas legalizadas surgen en la UGRA a principios de los años setenta con el desarrollo de las normas legales de entences, y; 
muy especialmente, con la promulgación por Decreto Ley del Reglamento de Instalaciones Nucleares y Radiactivas. Desde esa fecha hasta muy entrados los años ochenta, la protección radiológica la llevan personalmente los promotores de las instalaciones respectivas.

A mediados de los años ochenta, y tras crearse el Consejo de Seguridad Nuclear (CSN) y la Empresa Pública de Residuos Radiactivos (ENRESA), el CSN instó a la UGRA a unificar la gestión de residuos radiactivos de las distintas instalaciones, siendo esta la única función que se le exigía a la en materia de protección radiológica. La UGRA nombró una Coordinadora que se ocupó de realizar esta labor hasta mediados de los años noventa.

Tras la publicación del Real Decreto 1881/1991 (BOE 2-01-92) sobre utilización de Rayos X con fines de Diagnóstico Médico, la UGRA decidió crear una Unidad Técnica de Protección Radiológica (UTPR) Dental propia que asumiera las obligaciones impuestas por este Real Decreto. Simultáneamente, a la vista del aumento de instalaciones radiactivas, y en prevención del posible uso de material radiactivo por personas desaprensivas no adiestradas en protección radiológica (desconocedoras de las normas de legales correspondientes), se decidió crear un servicio centralizado que englobara las tareas de gestión de residuos existente, de asesoramiento y apoyo de las instalaciones legalmente constituidas, así como de vigilancia del uso irregular de sustancias radiactivas. Esta idea no tomó forma real hasta que en 1995 se promulgó la LPRL tras la cual la UGRA crea este Servicio de Protección Radiológica (SPR) y lo engloba en el GPCA.

\subsection{MISIONES DEL SERVICIO DE PROTECCIÓN RADIOLÓGICA}

El SPR tiene en la actualidad las siguientes misiones:

1. Asesoramiento en materia de protección radiológica al equipo de gobierno y demás órganos de la Universidad que lo soliciten.

2. Gestión de residuos radiactivos.

3. Asesoramiento y apoyo técnico a las instalaciones radiactivas de la Universidad.

4. Coordinación de supervisores responsables de las instalaciones en cuanto a temas comunes en protección radiológica.

5. Apoyo a la formación del personal mediante la promoción de cursos, libros, impresos, etc., de protección radiológica.

6. Prevención del uso ilegal de productos o emisiones radiactivas.

7. Control del uso legal de radiaciones ionizantes y vigilancia del cumplimiento de las normas y reglamentos en esta materia.

8. Prevención en general de la irradiación y contaminación de personas y medio ambiente en esta Universidad. 


\subsection{ACtuAciones}

Para el cumplimiento de las misiones expuestas se han realizado una serie de actuaciones entre las que destacamos las siguientes:

1. Promoción de un laboratorio ofertado a toda la comunidad universitario como "Servicio Técnico de Apoyo a la Investigación", donde los interesados encontrarán el personal e instalaciones adecuados y autorizados, así como el asesoramiento técnico necesario para realizar trabajos esporádicos con material radiactivo en condiciones de seguridad. Esta medida va encaminada a acabar con la piratería inconsciente y el uso ilegal de productos radiactivos fuera de instalaciones legalmente autorizadas.

2. Edición de un Boletín informativo dirigido a la comunidad universitaria.

3. Edición de señalizaciones y otras acciones puntuales de apoyo a las instalaciones.

4. Promoción del Servicio Médico Autorizado para los trabajadores en Instalaciones Radiactivas de la Universidad.

\subsection{ACTUACIONES FUTURAS}

Las actividades que está previsto poner en marcha de manera inmediata son las siguientes:

1. Control unificado de dosimetría personal.

2. Control de adquisición de material radiactivo.

3. Unificación de instalaciones radiactivas con control centralizado de la protección radiológica.

4. Programa de formación continua para el personal de las instalaciones a todos los niveles.

5. Comprobación y actualización de planes de emergencia.

6. Plan de previsiones técnicas periódicas de las instalaciones.

7. Edición de manuales, folletos,... de formación técnica.

\section{UNIDAD DE CALIDAD AMBIENTAL}

En abril de 1998, la UGRA, interesada por la mejora del medio ambiente; toma la decisión de aplicar en sus centros y servicios las directrices marcadas en la norma UNE-EN ISO 14001, con el objetivo de reducir el impacto que las actividades, docente, investigadora y de administración producen sobre el medio ambiente. 
Para ello, la UGRA firma un convenio con la empresa pública EGMASA para la implantación de un Sistema de Gestión Medioambiental (SGA) y la puesta a punto de Planes de Minimización de Residuos en todas sus instalaciones.

\subsection{Sistema DE GESTIÓN AMBIENTAL}

El Sistema de Gestión Medioambiental (SGA) a implantar se integrará en la gestión de todos los centros de la UGRA. Contendrá normas, manuales y guías, procedimientos de evaluación y auditorías, posibilitando la participación de su personal en todos sus niveles. El modelo de gestión medioambiental seleccionado está basado en la norma UNE-EN ISO 14001.

El proyecto para la implantación del SGA consta de las fases y etapas siguientes:

\section{Diagnóstico Ambiental.}

- Reunión con responsables y recopilación de normas.

- Análisis de información.

- Inspección ambiental "in situ" de las instalaciones

- Redacción del informe diagnóstico ambiental.

- Presentación de los resultados del diagnóstico ambiental.

2. Desarrollo de la documentación e implantación de procedimientos.

- Elaboración de la política ambiental.

- Diseño de la estructura del SGA.

- Relación de manuales del SGA.

- Elaboración de procedimientos.

- Adaptación de instrucciones técnicas de operaciones existentes.

- Elaboración de nuevas instrucciones técnicas de operación.

- Consolidación de la documentación del SGA.

\subsubsection{Diagnóstico ambiental}

El objetivo fundamental de esta fase del proyecto ha consistido en establecer el comportamiento de la UGRA con relación al medio ambiente.

La dificultad principal encontrada en la elaboración del diagnóstico medioambiental fue la variedad de actividades desarrolladas en los ámbitos docente e investigador, con impactos medioambientales muy diferentes.

El estudio y análisis realizados, basados en cuestionarios e inspecciones, permitió la clasificación de los centros en tres categorías según la incidencia medio- 
ambiental que presentan. En los centros caracterizados por una incidencia medioambiental alta (Facultad dé-Bellas Artes, Facultad de Ciencias, Facultad de Farmacia y Facultad de Medicina) se consideró necesario obtener información de los departamentos en ellos ubicados y proceder también a su clasificación.

Los aspectos evaluados fueron los siguientes:

- Emisiones controladas e incontroladas de contaminantes químicos a la atmósfera.

- Vertidos controlados e incontrolados a la red de saneamientos local.

- Producción y gestión de residuos peligrosos.

- Producción y gestión de residuos urbanos e inertes.

- Contaminación del suelo.

- Utilización de suelo, agua, combustibles y energía, así como otros recursos naturales.

- Emisiones de energía térmica, ruidos, olores, polvo, vibraciones e impacto visual.

- Grado de cumplimiento de la legislación medioambiental aplicable.

$\mathrm{Y}$, en general, el grado de adecuación a los requisitos exigidos por la norma UNE-EN ISO 14001.

De los aspectos señalados anteriormente, el más relevante es posiblemente la dificultad que presenta la gestión y el control de los residuos peligrosos que producimos. Ello se debe a tres razones fundamentalmente:

- La gran diversidad y tipología de residuos.

- Las pequeñas cantidades que de cada tipo se producen.

- La ausencia de uniformidad en las actividades realizadas.

Por otro lado, la gran variedad de actividades que se llevan a cabo en la UGRA, y en particular la existencia de instalaciones radiactivas, la utilización de animales para experimentación y el solapamiento con otras actividades de tipo sanitario, hace mucho más compleja la determinación del grado de cumplimiento de la legislación medioambiental de aplicación y la identificación de los aspectos medioambientales significativos.

La finalización de los trabajos correspondientes a esta fase culminaron con la elaboración y presentación en febrero del presente año de un informe en el cual se recogen también algunas medidas correctoras iniciales, no definitivas, pero necesarias en todo caso para la consecución de los objetivos del proyecto.

\subsubsection{Desarrollo del SGA}

La estructura documental del SGA de la UGRA consta de los siguientes documentos: 
- Manual del SGA.

- Manual de procedimientos.

- Manual de instrucciones técnicas.

- Registros del sistema.

Para elaborar estos documentos se constituyeron grupos mixtos formados por personal técnico y alumnos de Ciencias Ambientales, Químicas y Biológicas de la UGRA y personal técnico de EGMASA (*).

Dada la estructura organizativa de la UGRA, es necesario establecer varios niveles de gestión medioambiental, para se nombrarán responsables medioambientales en centros, servicios y departamentos.

Con objeto de ir implantando de manera progresiva los procedimientos, instrucciones y normas descritos en la documentación elaborada, se ha comenzado a mantener reuniones con personal de centros y departamentos.

\subsection{PLAN DE MINIMIZACIÓN}

El Plan de Minimización que se ha diseñado está basado en la elaboración de una serie de programas, cada uno de ellos orientado a minimizar un residuo, siguiendo un listado de prioridades basado en la incidencia medioambiental del residuo.

El plan consta de tres fases que comentamos brevemente a continuación.

\subsubsection{Estudio de fuentes de residuos}

Los datos obtenidos en el diagnóstico ambiental han permitido identificar las fuentes y sumideros de residuos.

\subsubsection{Diseño del plan de minimización}

Una vez identificados los residuos objeto de los programas de minimización, se procederá al diseño del plan mediante el desarrollo de las siguientes acciones:

(") Esta forma de trabajo ha servido para que alumnos de diferentes disciplinas se enfrenten con una situación real y diseñen, junto con personal técnico, las pautas medioambientales (guias, normas y procedimientos) que deberá seguir todo el personal de la UGRA. Por los resultados y por el aprendizaje de los alumnos, a los que una vez más agradecemos su colaboración, nos sentimos particularmente satisfechos de la experiencia. 
- Planteamiento de alternativas de actuación.

- Evaluación técnica de alternativas.

- Elección de opciones técnicamente viables.

- Análisis de viabilidad económica.

\subsubsection{Redacción y desarrollo de programas de minimización}

Tomando como base las decisiones del grupo de trabajo se elaborará un documento de síntesis en el cual se plantearán los programas unitarios de minimización de cada residuos.

\subsection{OTRAS ACTIVIDADES DE LA UNIDAD DE CALIDAD AMBIENTAL}

En 1996 la UGRA firmó un contrato con EGMASA para que esta empresa se encargase de la retirada y eliminación de los residuos peligrosos generados en los laboratorios como resultado de las actividades docente e investigadora.

Desde la creación del GPCA esta unidad coordina las tareas de envasado, etiquetado y almacenamiento de los residuos peligrosos hasta su retirada. Para el adecuado almacenamiento de los residuos hasta su retirada se ha financiado la construcción de dos locales, uno en la Facultad de Ciencias y otro en la Facultad de Farmacia, que incorporan todos los sistemas de seguridad precisos. Está prevista la construcción en el presente curso de otro local en la Facultad de Medicina.

En la tabla 4 se recogen las cantidades de residuos retiradas por la empresa EGMASA agrupados por categorías. Según se desprende de los datos de la tabla, en el periodo 1996-1998 se han recogido alrededor de $9.000 \mathrm{Kg}$ de residuos peligrosos. Este resultado, consecuencia del sistema de gestión de residuos implantado en nuestra Universidad, debemos verlo con optimismo, pero indica también que es necesario promover una cultura ambiental. Una mayor concienciación de nuestro personal docente e investigador en estos temas debe situar la generación de residuos peligrosos alrededor de 9.000 anuales.

Por otra parte, y en colaboración con el Vicerrectorado de Infraestructuras, a finales de 1998 se impulsó desde esta Unidad la instalación de contenedores por parte de la empresa INECUR, S.L. (Infraestructuras Ecológicas Urbanas) para la recogida gratuita de envases de aluminio y hojalata y de pilas botón en todos los centros y servicios de la UGRA y en otros puntos considerados estratégicos para este fin. Ello ha dado como resultado que en el periodo marzo-agosto del presente año se hayan recogido $865 \mathrm{Kg}$ de pilas botón y 14.600 envases de altiminio. 
Tabla 4. Producción de residuos peligrosos.

\begin{tabular}{|l|c|c|c|c|}
\hline \multirow{2}{*}{ CATEGORIA } & \multicolumn{4}{c|}{ CANTIDAD EN Kg } \\
\cline { 2 - 5 } & \multicolumn{1}{c|}{1996} & 1997 & $\mathbf{1 9 9 8}$ & TOTAL \\
\hline ÁCIDOS FUERTES Y DÉBILES & - & 125 & 175 & 300 \\
\hline BASES FUERTES Y DEBILES & - & 20 & 21 & 41 \\
\hline RESIDUOS ORGÁNICOS NO DISOLVENTES & - & 80 & 201 & 281 \\
\hline SALES & - & 15 & 125 & 140 \\
\hline ACEITE MINERAL & - & 120 & 65 & 185 \\
\hline METALES Y SUSTANCIAS SÓLIDAS & - & 95 & 535 & 630 \\
\hline DISOLVENTES' CLORADOS & - & 470 & 1.200 & 1.670 \\
\hline DISOLVENTES NO CLORADOS & - & 840 & 1.030 & 1.870 \\
\hline MERCURIO Y SUS DERIVADOS & - & 6 & 6 & 12 \\
\hline REACTIVOS DE LABORATORIO & 2.000 & 320 & 60 & 2.380 \\
\hline TETROXIDO DE OSMIO & - & 6 & 6 & 12 \\
\hline PILAS CONVENCIONALES & - & 1290 & 2 & 1.292 \\
\hline
\end{tabular}

